\title{
Association between PM10 and decrements in peak expiratory flow rates in children: reanalysis of data from five panel studies
}

\author{
G. Hoek*, D.W. Dockery+, A. Pope ${ }^{\ddagger}$ L. Neas ${ }^{+\#,}$ W. Roemer*, B. Brunekreef*
}

Association between PM10 and decrements in peak expiratory flow rates in children: reanalysis of data from five panel studies. G. Hoek, D.W. Dockery, A. Pope, L. Neas, W. Roemer, B. Brunekreef. @ERS Journals Ltd 1998.

ABSTRACT: Panel studies have found 1-3\% increases in reports of acute respiratory symptoms associated with each $10 \mu \mathrm{g} \cdot \mathrm{m}^{-3}$ increase in ambient concentrations of particles with a 50\% cut-off aerodynamic diameter of $10 \mu \mathrm{m}$ (PM10). Statistically significant decrements in population mean peak expiratory flow (PEF) have also been observed but their magnitude is only about $0.1 \%$ for the same exposure. This study evaluated whether this difference is due to the different measurement scales used for the expression of the effects of air pollution on PEF and respiratory symptoms.

The association between the prevalence of large decrements in PEF (more than $10 \%$ or $20 \%$ below the median) and PM10 concentrations was analysed using data from five recent panel studies. The effect estimates were compared to the effect estimates from original studies based on the population mean PEF.

The decrement in the population mean PEF for an increase of $10 \mu \mathrm{g} \cdot \mathrm{m}^{-3}$ of the same-day PM10 concentration was $0.07 \%$, averaged over all panels. A significant relative increase of $2.7 \%(95 \%$ confidence interval 1.6-3.8) in the prevalence of PEF decrements greater than $10 \%$ was associated with the same exposure.

In conclusion, defining the peak expiratory flow response by the prevalence of large decrements provides effect estimates of a comparable magnitude to effect estimates for the prevalence of reports of acute lower respiratory symptoms. Eur Respir J 1998; 11: 1307-1311.
*Dept of Environmental Sciences, Wageningen Agricultural University, Wageningen, The Netherlands. +Environment Epidemiology Program, Harvard School of Public Health, Boston, USA. DDept of Economics, Brigham Young University, Provo, USA. \#Channing Laboratory, Boston, USA.

Correspondence: G. Hoek, Dept of Environmental Sciences, Environmental and Occupational Health Unit, Wageningen Agricultural University, P.O. Box 238, 6700 AE Wageningen, The Netherlands, Fax: 31 317485278

Keywords: Air pollution, epidemiology, peak flow, PM10, respiratory

Received: September 291997

Accepted after revision February 281998

This work was performed during a Research Fellowship of The Netherlands Organization for Scientific Research to G. Hoek at the Harvard School of Public Health
Several recent panel studies have found significant incre-ases in acute respiratory symptoms associated with incre-ases in outdoor particle concentrations [1]. Summarized over the reviewed studies, the prevalence of lower respiratory symptoms and cough increased by 3 and $1.2 \%$, res-pectively, for an increase of $10 \mu \mathrm{g} \cdot \mathrm{m}^{-3}$ in the concentration of particles with a 50\% cut-off aerodynamic diameter of $10 \mu \mathrm{m}$ (PM10). In contrast, the mean percentage decrement of peak expiratory flow (PEF) associated with a 10 $\mu \mathrm{g} \cdot \mathrm{m}^{-3}$ increase in PM10 was only $0.08 \%$. Several more recent panel studies have reported similar results [2, 3].

There may be several explanations for the discrepancy between the magnitude of the symptom and PEF associations with particulate air pollution. Firstly, PEF may not be a sensitive lung function parameter. However, studies using full spirometry found only slightly larger percentage decrements for the forced expiratory volume in one second (FEV1). DOCKERY and Pope [1] reported a weighted mean FEV1 decrement of $0.15 \%$ associated with a $10 \mu \mathrm{g} \cdot \mathrm{m}^{-3}$ increase in the PM10 concentration. Secondly, the associations with subjective symptoms may be biased, although it is difficult to conceive a plausible mechanism that would cause similar bias in the different settings that have now been studied. A more likely hypothesis is that methods of data analysis for PEF have focused on average responses in a population, whereas symptom analyses focus on the fraction of the population reporting a specific (adverse) response. Small changes in the population mean PEF may be associated with more substantial changes in the occurrence of episodes of large PEF decrements. As-sociations between air pollution and the occurrence of large PEF decrements in panel studies have not been re-ported until now.

This report therefore describes the association between daily variations in PM10 concentration and the prevalence of episodes of large decrements in PEF. Data were reanalysed from five recent panel studies, which mostly involved children. The magnitude of the PM10 effect estimate was compared with the magnitude from the original analysis of the population mean PEF.

\section{Methods}

Data

Data were available from panel studies conducted in Utah Valley [4, 5], Bennekom, the Netherlands [6], Uniontown [2] and State College [3]. Detailed descriptions of the study populations and data collection methods are found in the original papers. In brief, daily measurements of evening PEF at home were conducted in all studies in panels of children. To characterize exposure, measurements of the ambient concentration of PM10 at fixed sites 
were available for all studies on a daily basis. The studies in Utah Valley and Bennekom were conducted in the winter and those in Uniontown and State College in the summer. Panels of children aged approximately 9-12 yrs were selected through the schools using a questionnaire on chronic respiratory symptoms. The panels in Uniontown, State College and the second winter in Utah Valley consisted of asymptomatic and symptomatic children (asthma diagnosed by a physician or reports of chronic cough or wheeze). Children using asthma medication were excluded from these three studies. In State College and Uniontown, data from the symptomatic and asymptomatic children were analysed together, as in the original papers $[2,3]$. The panels in Bennekom and the first winter in Utah Valley consisted of symptomatic children, including children using asthma medication. In the first winter in Utah an asthmatic patient panel was also recruited through local physicians, including both adults and children (age range $8-72 \mathrm{yrs}$ ). The use of asthma medication was high in this panel. Thus, data from seven panels were available. The original papers were followed with respect to restrictions on the subjects and days included in the analyses. A characterization of the panels is presented in table 1 .

\section{Data analysis}

In four of the original papers [2-5] associations between PEF and PM10 were estimated by linear regression of the daily population mean PEF deviation versus PM10 concentration. PEF deviations were calculated by subtracting subject-specific mean PEF values from the PEF measurement of a subject on a specific day. For each day the population mean deviation was calculated from the PEF measurements from all children contributing a valid measurement on that day. In one original paper individual linear regression analysis of PEF on PM10 concentration was conducted [6]. The mean of individual regression slopes was used to estimate the association between PEF and PM10. Both approaches thus focus on the mean response of the population. Several panel studies have documented that the two approaches yield similar effect estimates $[2,4,5]$. In all of the original papers, estimates of the effect of PM10 were adjusted for linear time trends and ambient temperature.

For the present study a different approach was taken. For each individual subject the median PEF was calculated. Percentage decrements were calculated for each measurement day for each subject by subtracting the individual median from the PEF measured on that day and dividing the difference by the median. The prevalence of decrements larger than 10 and 20\%, respectively, was calculated as the number of children demonstrating such a decrement divided by the total number of children reporting valid PEF measurements on each day of study. Transient decrements in FEV1 and forced vital capacity (FVC) larger than $10 \%$ have been considered as adverse effects $[7,8]$. Since the intra-individual variability of PEF is larger than that of FVC and FEV1, decrements of $20 \%$ were also analysed. Thus, this analysis focuses not on the mean response, but on the occurrence of biologically relevant events in the tail of the distribution.

Subtraction of the median was preferred to the mean because episodes of large PEF decrements affect the median less than the mean. Since lung growth occurs in children, the use of the highest PEF in the study (or the 95th percentile) was not appropriate. Most observations at the beginning of the study period were defined as a PEF decrement episode when maximum PEF was used.

The association between the prevalence of large PEF decrements and the PM10 concentration of the same day, previous day and the average of the previous 5 days (including the same day) was evaluated with logistic regression. Following the original studies, same-day temperature and a linear time trend were included as potential confounders. To adjust for autocorrelation in the residuals of this model, a first-order autoregressive model (AR-1) for the residuals was specified [9]. The residuals of the model were checked for remaining autocorrelation, using the partial autocorrelation function. In none of the panels were the residuals autocorrelated after specifying an AR-1 model. The specific logistic regression model incorporated an estimate of overdispersion or underdispersion into the standard errors of regression slopes. The overdispersion parameter ranged $0.75-1.35$, resulting in adjustments of the standard error of between -13 and $+16 \%$.

A statistical test of the homogeneity of the estimates of effects of the different panels was conducted using a chisquare test [10]. If this test suggested that effect estimates were homogeneous, weighted mean regression slopes were calculated from the panel-specific slopes using the inverse of the variance of the regression slopes as the weights. The standard error of the weighted mean was calculated as the square root of the inverse of the sum of the weights.

\section{Results}

\section{Data description}

A description of the study populations is presented in table 1. The studies in Utah Valley had the longest

Table 1. - Descriptive statistics of panel studies included in the reanalysis of peak flow data

\begin{tabular}{|c|c|c|c|c|c|}
\hline$\overline{\text { Panel }}$ & Number of days & Number of subjects & $\begin{array}{c}\text { Mean PEF } \\
\mathrm{L} \cdot \mathrm{min}^{-1}\end{array}$ & & $\begin{array}{l}\text { PEF } \\
\% \ddagger\end{array}$ \\
\hline Utah Valley, school* & 107 & 34 & $260(149-403)$ & 10 & $(3-22)$ \\
\hline Utah Valley, patient* & 107 & 21 & $320(109-534)$ & 12 & $(3-37)$ \\
\hline Utah Valley, symptomatic ${ }^{\dagger}$ & 100 & 39 & $298(187-403)$ & 9 & $(2-28)$ \\
\hline Utah Valley, asymptomatic $^{\dagger}$ & 100 & 40 & $308(203-424)$ & 7 & $(2-15)$ \\
\hline Bennekom & 83 & 67 & $298(119-434)$ & 9 & $(3-25)$ \\
\hline Uniontown & 74 & 83 & $331(222-450)$ & 6 & $(2-17)$ \\
\hline State College & 53 & 108 & $342(237-463)$ & 5 & $(1-12)$ \\
\hline
\end{tabular}

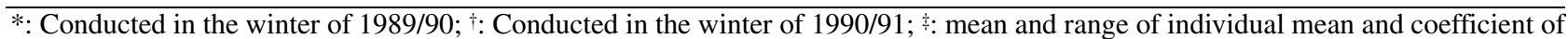
variation $(\mathrm{CV})$ (SD divided by mean) of peak expiratory flow (PEF). 
follow-up, while those in Uniontown and State College had the largest number of subjects. The mean coefficient of variation $(\mathrm{CV})$ of the panels reflects the composition of the panels. The asymptomatic panel in Utah Valley and the panels from Uniontown and State College included the largest percentage of children without chronic respiratory symptoms. The four panels from Utah and the panel from Bennekom were studied in the winter, and the panels in Uniontown and State College in the summer.

The distribution of PM10 concentrations in the respective study periods is shown in table 2 . PM10 concentrations in the three winter panel studies (involving five panels) exceeded $100 \mu \mathrm{g} \cdot \mathrm{m}^{-3}$ between 6 and 29 days. In the two summer panels all PM10 concentrations were below 85 $\mu \mathrm{g} \cdot \mathrm{m}^{-3}$.

The mean prevalence of $10 \%$ decrements in PEF ranged from $4 \%$ for the State College panel to $17 \%$ for the

Table 2. - Distribution of daily average PM10 concentrations $\left(\mu \mathrm{g} \cdot \mathrm{m}^{-3}\right)$ in the respective study periods

\begin{tabular}{lrcccc}
\hline Panel & Min. & Med. & $\begin{array}{c}\text { 95th } \\
\text { percentile }\end{array}$ & Max. & $\begin{array}{c}\text { No. of } \\
\text { days }\end{array}$ \\
\hline Utah Valley, winter & 11 & 35 & 101 & 195 & 107 \\
$\quad$ 1989/90* & & & & & \\
Utah Valley, winter & 7 & 62 & 189 & 251 & 100 \\
$\quad$ 1990/91 & & & & & \\
Bennekom & 13 & 54 & 133 & 175 & 79 \\
Uniontown & 7 & 34 & 65 & 83 & 74 \\
State College & 10 & 29 & 68 & 83 & 52 \\
\hline
\end{tabular}

Min.: minimum; Med.: median; Max.: maximum; No.: number; *: school and patient panel studied in this winter; ${ }^{\dagger}$ : symptomatic and asymptomatic panel studied in this winter. PM10: particles with a $50 \%$ cut-off aerodynamic diameter of $10 \mu \mathrm{m}$.

Table 3. - Daily prevalence (\%) of 10 and $20 \%$ percentage decrements in evening peak expiratory flow (PEF) below individual median PEF

\begin{tabular}{lrlll}
\hline Panel & \multicolumn{2}{c}{$\begin{array}{c}10 \% \\
\text { decrement }\end{array}$} & \multicolumn{2}{c}{$\begin{array}{c}20 \% \\
\text { decrement }\end{array}$} \\
\hline Utah Valley, school & 14 & $(0-39)$ & 5 & $(0-19)$ \\
Utah Valley, patient & 17 & $(0-53)$ & 6 & $(0-22)$ \\
Utah Valley, symptomatic & 10 & $(0-28)$ & 4 & $(0-13)$ \\
Utah Valley, asymptomatic & 8 & $(0-21)$ & 2 & $(0-12)$ \\
Bennekom & 11 & $(3-21)$ & 3 & $(0-11)$ \\
Uniontown & 8 & $(0-27)$ & 2 & $(0-9)$ \\
State College & 4 & $(0-9)$ & 1 & $(0-5)$
\end{tabular}

Values are shown as mean with range in parentheses.
Utah patient panel (table 3 ). These mean prevalences partly reflect the different percentages of asthmatic subjects in the different panels. Seasonal variation may explain some of the differences between summer and winter panels.

Associations of PM10 with prevalence of large decrements in peak expiratory flow

Increases in the same-day PM10 concentration of 10 $\mu \mathrm{g} \cdot \mathrm{m}^{-3}$ were associated with increases in the prevalence of $10 \%$ PEF decrements in all panels (table 4). A chi-square test did not show significant heterogeneity among effect estimates from the individual panels. The combined effect estimate, calculated as $100 \times(1-\exp ($ combined $\beta \times 10))$, was $2.7 \%$ (95\% confidence interval (CI) 1.6-3.8). This implies that the prevalence of decrements in PEF $>10 \%$ increased for example, from 10 to $10.27 \%$ with each $10 \mu \mathrm{g} \cdot \mathrm{m}^{-3}$ increase in the PM10 concentration. Slightly larger increases were found for $20 \%$ PEF decrements, but the precision was lower because of the low prevalence of such large decrements. The estimates for the two summer panels (Uniontown and State College) were less precise because of the smaller number of days of study, the smaller variation in PM10 concentration and the lower prevalence of large PEF decrements.

The combined effect estimates for the previous-day PM10 concentration were similar to the estimates for same-day PM10: 2.4\% (95\% CI 1.3-3.5) and 3.4\% (95\% CI 1.8-5.0) for PEF decrements of 10 and $20 \%$, respectively. The five-day average PM10 concentration was associated with stronger increases in the prevalence of 10 and 20\% PEF decrements. This was especially the case for the two panels in the first winter in Utah Valley and the Bennekom (10\% decrements) panel. The difference in the strength of association between same-day and five-day average PM10 found for the Utah patient panel is consistent with the results of polynomial distributed lag models in the original paper showing significant associations after lag two only [4]. This pattern may be explained by the use of extra asthma medication mitigating initial but not later peak flow responses to air pollution [4].

The difference between magnitude of same-day and five-day average PM10 associations is partly explained by the lower variability of the latter. The variability of the five-day average (characterized by the interquartile range,

Table 4. - Change in prevalence of 10 and $20 \%$ peak expiratory flow (PEF) decrements with an increase of $10 \mu \mathrm{g} \cdot \mathrm{m}^{-3}$ in the daily average $\mathrm{PM}_{10}$ concentration, adjusted for time trend and ambient temperature

\begin{tabular}{|c|c|c|c|c|c|c|c|c|}
\hline \multirow[b]{2}{*}{ Panel } & \multicolumn{4}{|c|}{ Same-day PM10 } & \multicolumn{4}{|c|}{ Five-day average PM10 } \\
\hline & \multicolumn{2}{|r|}{$>10 \%$} & \multicolumn{2}{|r|}{$>20 \%$} & \multicolumn{2}{|r|}{$>10 \%$} & \multicolumn{2}{|r|}{$>20 \%$} \\
\hline Utah Valley, school & 6.1 & $(3.2-9.1)$ & 3.4 & $(-0.9-7.9)$ & 8.6 & $(4.4-12.9)$ & 7.7 & $(1.5-14.3)$ \\
\hline Utah Valley, patient & 0.6 & $(-2.7-4.0)$ & 0.2 & $(-4.5-5.1)$ & 3.6 & $(-1.1-8.5)$ & 3.2 & $(-3.5-10.4)$ \\
\hline Utah Valley, symptomatic & 2.1 & $(0.3-3.9)$ & 3.5 & $(1.2-5.9)$ & 3.0 & $(1.0-5.1)$ & 4.0 & $(1.2-6.8)$ \\
\hline Utah Valley, asymptomatic & 2.6 & $(0.6-4.6)$ & 2.6 & $(-1.2-6.6)$ & 3.9 & $(1.4-6.3)$ & 3.0 & $(-1.8-7.9)$ \\
\hline Bennekom & 0.8 & $(-3.4-5.2)$ & 8.7 & $(2.3-15.4)$ & 6.9 & $(-1.4-15.8)$ & 8.4 & $(-4.4-22.9)$ \\
\hline Uniontown & 12.4 & $(0.7-25.5)$ & 21.4 & $(-2.3-50.8)$ & 6.3 & $(-11.2-27.2)$ & 34.6 & $(-6.1-92.9)$ \\
\hline State College & 4.9 & $(-12.3-25.5)$ & -2.5 & $(-35.7-48.0)$ & 18.7 & $(-6.5-50.7)$ & 21.6 & $(-29.3-109)$ \\
\hline p-value heterogeneity & 0.10 & & 0.36 & & 0.30 & & 0.66 & \\
\hline Combined data & 2.7 & $(1.6-3.8)$ & 3.4 & $(1.8-5.0)$ & 4.2 & $(2.7-5.5)$ & 4.4 & $(2.3-6.5)$ \\
\hline
\end{tabular}

Values are percentage change with $95 \%$ confidence interval in parentheses. Percentage changes were calculated as 100×(1- OR), with OR (odds ratio) calculated as exp(combined $\beta \times 10)$. PM10: particles with a $50 \%$ cut-off aerodynamic diameter of $10 \mu \mathrm{m}$. 
Table 5. - Same-day PM10 associations of population mean peak expiratory flow (PEF) and prevalence of $10 \%$ PEF decrements, adjusted for time trend and ambient temperature

\begin{tabular}{llcc}
\hline Panel & \multicolumn{1}{c}{$\begin{array}{c}\text { Population } \\
\text { mean PEF }\end{array}$} & \multicolumn{2}{c}{$\begin{array}{c}\text { Prevalence of } \\
10 \% \text { PEF } \\
\text { decrements }\end{array}$} \\
\hline Utah Valley, school & $-0.25(-0.39--0.10)^{*}$ & 6.1 & $(3.2-9.1)^{\dagger}$ \\
Utah Valley, patient & $-0.05(-0.22-0.13)$ & 0.6 & $(-2.7-4.0)$ \\
$\begin{array}{l}\text { Utah Valley, } \\
\text { symptomatic }\end{array}$ & $-0.06(-0.12-0.00)$ & 2.1 & $(0.3-3.9)$ \\
Utah Valley, & $-0.04(-0.09-0.02)$ & 2.6 & $(0.6-4.6)$ \\
asymptomatic & & & \\
Bennekom & $-0.09(-0.20-0.01)$ & 0.8 & $(-3.4-5.2)$ \\
Uniontown & $-0.19(-0.35--0.03)$ & 12.4 & $(0.7-25.5)$ \\
State College & $-0.03(-0.18-0.11)$ & 4.9 & $(-12.3-25.5)$ \\
Combined data & $-0.07(-0.10--0.03)$ & 2.7 & $(1.6-3.8)$ \\
\hline
\end{tabular}

*: per cent change in mean PEF (95\% confidence interval (CI) in parentheses) associated with a $10 \mu \mathrm{g} \cdot \mathrm{m}^{-3}$ change in PM10 concentration, calculated from regression slopes in original papers and mean PEF of the panel. $\%$ : per cent change in prevalence of $10 \%$ PEF decrements (95\% CI in parentheses) associated with a $10 \mu \mathrm{g} \cdot \mathrm{m}^{-3}$ change in PM10 concentration. PM10: particles with a $50 \%$ cut-off aerodynamic diameter of $10 \mu \mathrm{m}$.

IQR) was on average $80 \%$ of the variability of the sameday PM10 concentration. For the five winter panels the ratio of the five-day mean to the same-day PM10 IQR was larger (between 0.85-0.92) than for the two summer panels $(0.55-0.67)$.

\section{Comparison with population mean analysis}

A comparison with effect estimates reported in the original papers is presented in table 5. For all evaluated panel studies decrements in the population mean PEF with increasing same-day PM10 concentration were reported. The combined decrement of the population mean PEF for an increase in the PM10 concentration of $10 \mu \mathrm{g} \cdot \mathrm{m}^{-3}$ was $0.07 \%$. The largest negative slopes for the population mean PEF were found for the school panel in Utah Valley and the panel in Uniontown, PA, consistent with the order of the effect estimates in the $10 \%$ PEF decrement analysis.

\section{Discussion}

The main findings of this study were that increases in the PM10 concentration with $10 \mu \mathrm{g} \cdot \mathrm{m}^{-3}$ were associated with an increase in the prevalence of $10 \%$ PEF decrements of $2.7 \%$. The same PM10 concentration increase resulted in an average decrement of the population mean PEF of $0.07 \%$. The difference between the magnitude of $\mathrm{PEF}$ and symptom effect estimates for PM10 in previous panel studies is thus largely due to the different measurement scales used in the original analyses.

The combined slope of $0.07 \%$ PEF decrement per 10 $\mu \mathrm{g} \cdot \mathrm{m}^{-3} \mathrm{PM} 10$ predicts a decrement of only $0.7 \%$ of the population mean PEF during a typical episode with an increase of the PM10 concentration of $100 \mu \mathrm{g} \cdot \mathrm{m}^{-3}$. This small decrement in PEF would be interpreted only as a mild response. In contrast, the combined estimate for the same panel studies indicates that the prevalence of $10 \%$ or larger PEF decrements would increase by $31 \%$ during such an episode. Transient decrements in FVC and FEV1 of $10 \%$ have been considered as the border between mild and moderate responses $[7,8]$. Thus, a considerable increase in the number of subjects with a response that could be characterized as adverse is associated with increased PM10 concentrations. The magnitude of the PEF effect estimate is comparable to the effect estimate reported for the increase in the prevalence of lower respiratory symptoms (LRS) [1]. The combined effect estimate in that review was an increase of $3 \%$ in the prevalence of LRS, with an increase in the PM10 concentration of $10 \mu \mathrm{g} \cdot \mathrm{m}^{-3}$. The finding of adverse effects on the more objective PEF measurements supports the report of adverse symptom responses in panel studies, and adds to the database of documented adverse effects of ambient particulate matter, including increased hospital admissions and mortality [1].

The larger effect estimate found for the prevalence of large PEF decrements can be due to different mechanisms. One possibility is that a small shift in the mean of the distribution may lead to larger changes of the probability of responses in the tail of the distribution. This hypothesis is supported by the fact that increases in PEF of 10 and 20\% above the individual median were negatively correlated with the PM10 concentration.

An increase of $10 \mu \mathrm{g} \cdot \mathrm{m}^{-3}$ of same-day PM10 concentration was associated with a $-1.0 \%(95 \% \mathrm{CI}-2.5-0.5)$ and $-7.8 \%$ (95\% CI -11.5--3.9) change in the prevalence of increases of PEF of 10 and $20 \%$, respectively.

Another possibility is that only a few children responded on a specific day with a large decrement, while the vast majority of the children did not respond at all. This hypothesis was tested by repeating the original population mean PEF analysis after excluding the children with the largest fraction of days with a $10 \%$ PEF decrement (children above the 75th percentile of the distribution of childspecific fractions of $10 \%$ PEF decrement days from each panel). Since most children reported a $10 \%$ PEF decrement on at least one day, it was not possible to exclude all children who reported these decrements once. The combined effect estimate of $-0.07 \%$ (SE $0.02 \%$ ) was only $8 \%$ lower than the combined effect estimate using all data (table 5). The combined slope remained significantly different from zero. The small change in the combined slope was due to a small increase in the slope for the symptomatic and asymptomatic Utah panels (panels with a large weight). The average slope from the other five panels decreased from $-0.11 \%$ to $-0.07 \%$, but remained highly significant.

The original population mean PEF analysis was repeated after excluding all days on which a $10 \%$ PEF decrement was reported. The combined effect estimate of $-0.06 \%$ (SE $0.01 \%$ ) was $23 \%$ lower than the combined effect estimate using all data (table 5).

The finding of a significant decrease in the prevalence of 10 and $20 \%$ PEF increases with higher PM10 concentrations suggests that a shift in the distribution largely explains the larger effect estimates in this study. The effect of PM10 on the population mean PEF remained after excluding the $25 \%$ of children with the highest percentage of days with $10 \%$ PEF decrements, thus showing that a shift in the response of the whole population occurs with increasing PM10 concentration. The small mean response was, therefore, not caused by large PEF decrements in a 
few children accompanied by a majority of nonresponding children. This continuity of response is analogous to observations about population distributions of characteristics such as blood pressure and body weight. Populations with a high value for central tendency also tend to have a high fraction of subjects with an "extreme" value [11]. Rose [11] presents several examples in which small changes in the mean of a variable result in a substantial decrement in the number of subjects with extreme, biologically relevant values. A 3\% fall in the average systolic blood pressure was associated with a $25 \%$ decrement of subjects with high blood pressure in UK adults [11]. This phenomenon can easily be illustrated from the normal distribution. In a normal distribution with a coefficient of variation (sD/ mean) of $10 \%$, a change in the mean of $0.07 \%$ predicts increases of $1.1 \%$ and $1.6 \%$ in the probability of decrements of 10 and $20 \%$ ( 1 and 2 SD, respectively). Similar findings were obtained in a recent cross-sectional study in 24 North American cities. A 3.5\% population mean decrement of FVC was associated with a $52 \mathrm{nmol} \cdot \mathrm{m}^{-3}$ difference in strongly acidic particles [12]. Such a difference would not be considered clinically important. However, an odds ratio of 2.5 was found for low lung function (defined as $<85 \%$ of predicted) associated with the same exposure in that study, illustrating that the percentage of children with clinically relevant low lung function was clearly increased in the more polluted communities.

The discussion in the previous paragraph suggests that the two different methods of analysis do not present unrelated effect estimates. It is unlikely for a specific panel that the mean response would be close to zero while a significant increase in the number of children with $10 \% \mathrm{PEF}$ decrements occurs with increasing air pollution. This is supported to some extent by the comparison of effect estimates in the panels included in this report (table 5). In both methods of analysis, the largest effect estimates were obtained for the Utah school panel and the Uniontown panel. The small variability in effect estimates across studies (which could be due to random error alone) limits the possibility of drawing firm conclusions from these panel studies. It is therefore of interest to repeat this comparison with a larger range of effect estimates.

For this study we have chosen to calculate PEF decrements compared with the individual median PEF. In shorter studies on adults, with a smaller time trend, deviations from the maximum (or a more robust measure such as the 95th percentile) in the study period may be used. In longer studies on children, with a strong time trend due to lung growth, it may be necessary to split the study period into different parts to prevent all observations in the first weeks of the study period being considered as a large PEF decrement.

In conclusion, the large discrepancy between the small magnitude of peak expiratory flow and the more substantial concentration of particles with a $50 \%$ cut-off aerody- namic diameter of $10 \mu \mathrm{m}$, effect estimates on respiratory symptoms in previous panel studies, which is largely due to the different measurement scale of both variables. When peak expiratory flow is analysed in a similar way to symptoms by modelling the prevalence of large peak expiratory flow decrements, the estimates of the effects of the concentration of particles with a 50\% cut-off aerodynamic diameter of $10 \mu \mathrm{m}$ on lower respiratory symptoms and peak expiratory flow are similar. The effects observed on peak expiratory flow and on acute respiratory symptoms are therefore much more coherent than was apparent from the earlier reported analyses of these data.

\section{References}

1. Dockery DW, Pope CA III. Acute respiratory effects of particulate air pollution. Annu Rev Public Health 1994; 15: 107-132.

2. Neas LM, Dockery DW, Koutrakis P, Tollerud DJ, Speizer FE. The association of ambient air pollution with twice daily peak expiratory flow rate measurements in children. Am J Epidemiol 1995; 141: 111-122.

3. Neas LM, Dockery DW, Burge H, Koutrakis P, Speizer FE. Fungus spores, air pollutants and other determinants of peak expiratory flow rate in children. Am J Epidemiol 1996; 143: 797-807.

4. $\quad$ Pope CA III, Dockery DW, Spengler JD, Raizenne ME. Respiratory health and PM10 pollution: a daily time series analysis. Am Rev Respir Dis 1991; 144: 668-674.

5. Pope CA III, Dockery DW. Acute health effects of PM10 pollution on symptomatic and asymptomatic children. Am Rev Respir Dis 1992; 145: 1123-1128.

6. Roemer W, Hoek G, Brunekreef B. Effect of ambient winter air pollution on respiratory health of children with chronic respiratory symptoms. Am Rev Respir Dis 1993; 147: 118-124.

7. Lippmann M. Health significance of pulmonary function responses to airborne irritants. J Air Poll Control Assoc 1988; 38: 881-887.

8. World Health Organization. Acute effects on health of smog episodes. Geneva, WHO Regional Publications, European Series, No. 43, 1992.

9. Schwartz J, Dockery DW, Neas LM, et al. Acute effects of summer air pollution on respiratory symptom reporting in children. Am Rev Respir Dis 1994; 150: 1234 1242.

10. DerSimonian R, Laird N. Meta-analysis in clinical trials. Controlled Clin Trials 1986; 7: 177-188.

11. Rose G. Some implications of population change. In: The Strategy of Preventive Medicine. Oxford, Oxford University Press, 1992; pp. 64-94.

12. Raizenne M, Neas LM, Damokosh AI, et al. Health effects of acid aerosols on North American children: pulmonary function. Environ Health Perspect 1996; 104: 506-514. 